\title{
Attacker profiling in quantitative security assessment based on attack trees
}

\author{
Aleksandr Lenin ${ }^{1,2}$, Jan Willemson ${ }^{1}$ and Dyan Permata Sari ${ }^{2} *$ \\ 1 Cybernetica AS \\ 2 Tallinn University of Technology
}

\begin{abstract}
Providing meaningful estimations for the quantitative annotations on the steps of complex multi-step attacks is hard, as they are jointly influenced by the infrastructure and attacker properties. The paper introduces attacker profiling as a concept of separation of the infrastructure properties from the properties of malicious agents undertaking strategic decisions in the considered environment. We show that attacker profiling may be integrated into existing quantitative security assessment tools without any significant performance penalty. As an example of such integration we introduce the new analysis tool named ApproxTree+ which is an extension of the existing ApproxTree tool, enhancing it by incorporating attacker profiling capabilities into it.
\end{abstract}

\section{Introduction}

Targeted malicious attacks are intentional by their nature and may be interpreted as sequences of actions (attack steps) performed by malicious agents undertaking informed strategic decisions in the target infrastructure. This way we can distinguish between the two landscapes - the one which we call the threat landscape and the vulnerability landscape. The threat landscape is formed by various kinds of malicious agents - they have different sets of properties, available resources, varying intentions, motivations, views, and expectations of the target infrastructure. These properties determine strategic preferences of the agents, and eventually their behavior. The vulnerability landscape is formed by the infrastructure of the organization, its employees, assets, policies, processes, etc. Both landscapes are dynamic by their nature and are constantly changing. The threat landscape may change due to the agent behavior (e.g. increase in resources available to the agent) as well as external events,

\footnotetext{
* The research leading to these results has received funding from the European Union Seventh Framework Programme (FP7/2007-2013) under grant agreement ICT-318003 (TRE $\mathrm{SPASS}$ ). This publication reflects only the authors' views and the Union is not liable for any use that may be made of the information contained herein.
} 
while the vulnerability landscape may change due to the infrastructure updates (e.g. patching, component replacement, awareness training, deployment of defensive measures, etc.) as well as unintentional events.

We propose the separation between the infrastructure properties (the vulnerability landscape) and the adversarial properties (the threat landscape), represented by an attacker profile. This separation adds flexibility to the quantitative security analysis enabling the assessment of operational security risks using different combinations of attacker profiles and infrastructure properties providing much deeper insight on the surrounding risk landscape. Besides, attacker profiling increases the reliability of the analysis results as the separation of infrastructure properties and attacker properties allows to update these values in a timely manner independently from each other and reflect the ever changing risk landscape in a more reliable way.

The paper aims at introducing attacker profiling in the context of quantitative security analysis based on attack trees and demonstrates integration of attacker profiling into existing security assessment tools introducing the new tool named ApproxTree+. In the introduced ApproxTree+ model the considered infrastructure properties (cost, difficulty, minimal required attack time) are quantitative annotations on the attack tree leaves, while the adversarial properties (budget, skill, available time) are described by attacker profiles. Additionally we compare the performance of the profiling computations to the ApproxTree approach [1] and reassess if the genetic algorithm parameters, used by ApproxTree for fast approximations, are optimal for the profiling computations.

The outline of the paper is the following: Section 2 outlines the state of the art in quantitative security assessment, attack trees, and attacker profiling. Section 3 describes motivation for the attacker profiling in security risk assessment. Section 4 introduces the ApproxTree+ tool, while Section 5 outlines the tool performance analysis results. Section 6 briefly lists the achievements made so far and outlines areas for future research.

\section{Related Work}

\section{$2.1 \quad$ Attack trees}

Attack trees as one of the ways of quantitative security assessment, evolved from fault trees [2] and were popularized by Schneier [3] who suggested to use them as a way to model security threats and to perform quantitative security assessment using this convenient hierarchical representation by means of bottom-up single parameter propagation. Quantitative security 
assessment has been studied by various researchers [4-8] and different variations of techniques and methodologies were suggested.

Buldas et al. [9] suggested to use multi-parameter approach instead of the historical single-parameter one and applied economic reasoning by propagating adversarial utility. This kind of analysis allowed to assess whether the analyzed system is secure against targeted rational profitoriented attacks.

Jürgenson and Willemson improved the model of Buldas et al., making their parallel [10] and serial [11] models consistent with Mauw and Oostijk foundations [12] and introducing genetic approach to speed up computations. The parallel model assumed that the attacker launches attack steps, required to fulfil the attack scenario, simultaneously, while the serial model assumed that an attacker launches the attack steps in a predefined order.

Later, Buldas and Stepanenko introduced the failure-free model [13] suggesting not to limit the adversary in any way and thus analyzing fully adaptive adversarial utility upper bounds. This approach was later improved by Buldas and Lenin in [14]. Their model better conforms to the upper bounds ideology and is computationally less complex.

For a more thorough overview of the quantitative security analysis using attack trees we refer the reader to [15].

\subsection{Attacker profiling}

Back in 1998, Philips et al. [16] outlined the importance of the attacker feature in attack graphs for network vulnerability analysis. Several research projects have focused on attacker profiling using honeypot in "Know Your Enemies" series [17-19] which outlined the range of techniques and tools that were used by attackers for reconnaissance and also motives of the blackhat community. Several researchers proposed the concept of attacker personas, which was related to goal, motivations, attitudes, and skills [20-23]. Shamal et al. highlighted insider threat motivations and characteristics, as well as the use of attacker personas for threat identification. Collective or individual attacker has been the main interest for several research efforts [24-28], and in these models attacker personas were heavily related to attacker skill, motivation, ideology, and goal.

Harold et al. [29] mentioned the importance of attacker characteristics and also the complexity of the attacks assessing risks of an e-voting system. The authors argue that the likelihood of attacks can be referred to as cost of an attacker, which can be estimated on various scales and measured in various units, such as dollars, number of attackers, time 
invested into attacking, and effort. In addition, Sallhammar et al. [30] demonstrate the process of deriving the probability of the expected attacker behavior in assumption that the attacker has complete information about the vulnerabilities of the targeted systems. Tipton et al. [31] argue that risk aversion, degree of difficulty, discoverability, ease of access, effectiveness of controls, effort, incentive, interest, skill level, motivation, resources required, risk of detection, and special equipment needed are the fagents that can be included in attacker profiling. There are some common parameters that are most often used in research projects to define an attacker profile - these values are more feasible for quantitative analysis and give clear understanding of attacker properties.

\subsection{Parallel model}

The parallel model [10] by Jürgenson et al. allows to assess whether the analyzed system is secure against targeted rational profit-oriented attacks by assessing adversarial utility. In case the utility is positive, the system is considered to be insecure, as profitable attack vectors which may result in positive outcome for an attacker are likely to exist. Otherwise the analysis assumed that the system is reasonably secure to withstand emerging attacks.

An attack scenario, represented by an attack tree, is treated as a monotone Boolean function, each variable of which corresponds to a leaf node in the attack tree, and logic operators correspond to the refined nodes in the attack tree. The successful outcome of an elementary attack is modelled by assigning value 1 to the corresponding variable in the Boolean function. If the Boolean function is satisfied, the attacker has succeeded in the security scenario. More complex multi-step attacks are modelled as attack suites.

The computational method maximizes the adversarial utility over the entire set of satisfying attack suites. The complexity of the approach arises from the need to process the entire set of $2^{n}$ attack suites, which introduces unnecessary overhead. Even with the optimizations proposed in [10] this approach was able to analyze attack trees of at most 20 leaves in reasonable time which has made this method inapplicable for the practical case analysis.

To overcome limitations of the parallel model [10], a set of further optimizations was proposed by Jürgenson et al. in [1] and implemented in the tool later called ApproxTree.

More significant contribution of the paper is the development of genetic algorithm for fast approximations, which increased performance 
compared to [10]. The implementation of the approach described in the paper reached $89 \%$ confidence $^{1}$ level within 2 seconds of computation for the tree having up to 29 leaves. As the genetic algorithm is very scalable it has potential to be used for the analysis of practical attack trees containing more than 100 leaves. The computational complexity of the suggested approximation algorithm in the worst case was estimated to be $\mathcal{O}\left(n^{4}\right)$. The authors have performed benchmarking tests and experimentally derived the optimal set of values for genetic algorithm parameters.

\section{Motivation for the attacker profiling}

An attack tree is a hierarchical description of possible attacks against the target infrastructure. Constructing an attack tree, analysts include all possible attack scenarios in the tree. Some of them are more realistic, some are less, considering the environment in which such a system is deployed. This way, attack tree analysis assumes an overpowered attacker who is capable of launching every possible attack, included in the attack tree, against the system. However, real life attacks are, as a rule, not so powerful and thus analysis assuming the almighty adversary concept does not provide deep insight on the security risks taking into account the surrounding risk landscape. Applying attacker profile to the attack tree invalidates certain nodes and eventually entire subtrees in the initial attack tree, thus enabling the independent analysis of the derived attack scenarios, containing attacks feasible for the considered class of malicious agents. Depending on the severity of adversarial limitations used in the profile, the derived attack scenario may be much smaller and thus much easier to analyze.

Quantitative security analysis relies on quantitative annotations (e.g. likelihood of success in an attack step, time required to launch an attack step, etc.) assigned to single attack steps in complex multi-step attacks. We believe that the quantitative metrics of these annotations is jointly influenced by various sets of underlying components in threat- as well as vulnerability landscapes. Thus it is rather difficult to provide a trustworthy and reliable quantitative estimation for such parameters as it is practically impossible to estimate the cumulative effect of several underlying factors altogether. Such kind of joint estimations are, as a rule, imprecise and contain reasonable degree of uncertainty.

\footnotetext{
${ }^{1}$ By confidence authors mean the ratio of the trees actually computed correctly by the suggested approximation technique, compared to the precise outcome.
} 
For example, it is almost impossible to provide a meaningful estimation for the time parameter, as the time, required for an attack step, depends on the attacker skills, capabilities, available resources, previous experience, etc. (agent properties), as well as on the difficulty of the attack step itself (infrastructure property). Similarly, the likelihood of success depends on attacker skill, difficulty of the attack step, and time invested into attacking. The more skilful and experienced the attacker is, the more likely he is to succeed in an attack step. The more resources are available to the attacker, the more likely will he be successful in an attack step. Similar reasoning may be applied to the skill parameter - the more experienced the attacker is, the less difficult is the process for him, the less time it will take to succeed in an attack step. Less skilled attacker, given sufficient time, may be as efficient (in terms of likelihood of success) as a more skilled attacker who has less time for attacking. Similar logic may be applied to other parameters as well.

Despite that, the analysis has to deal somehow with the ever changing nature of each of the landscapes mentioned above and update (or reassess) the estimations of the corresponding quantitative annotations in a timely manner. It is unclear how to update such joint estimations in case some of its components change while the others remain unchanged, or, on the contrary, when all its components change.

In order to tackle the difficulties outlined above the propose attacker profiling as a step forward in dealing with the challenges of security metrics.

\section{The ApproxTree+ model}

We introduce the ApproxTree+ model - the new model for quantitative assessment of operational security risks. The computational method is built on the logic of the parallel attack tree model [10] and fast approximations of ApproxTree [1], improved by adding attacker profiling considerations into the method.

\subsection{Definitions}

Definition 1 (Attack Suite). Attack suite $\sigma$ is a set of elementary attacks which have been chosen by the attacker to be launched and used to try to achieve the attacker goal.

Definition 2 (Satisfying attack suite). A satisfying attack suite $\sigma$ evaluates the Boolean function of the attack scenario to true when all 
the elementary attacks from the attack suite $\sigma$ have been tried and have been evaluated to true and false values, correspondingly, if the elementary attack was successful or failed.

Definition 3 (Attacker profile). An attacker profile is a set of characteristics and properties uniquely describing the attacker under consideration:

1. Budget - the monetary resource of the attacker, measured in currency units.

2. Proficiency - the skill level of the attacker, measured on an ordinal scale (Low/Medium/High).

3. Time - the available time resource of the attacker, measured on an ordinal scale (Seconds/Minutes/Hours/Days).

Definition 4 (Attacker profile). An attacker profile is a function $\mathcal{P}_{f}(\sigma)$ which takes an attack suite $\sigma$ as input and returns true, iff the considered attacker is capable of launching all the attacks in the attack suite, and false otherwise. The attacker is capable of launching an attack suite $\sigma=\left\{\mathcal{X}_{1}, \mathcal{X}_{2}, \ldots, \mathcal{X}_{n}\right\}$, if:

1. $\mathcal{P}_{\text {budget }} \geqslant \sum_{i=1}^{n} \operatorname{Cost}\left(\mathcal{X}_{i}\right)$,

2. $\forall \mathcal{X}_{i} \in \sigma: \mathcal{P}_{\text {skill }} \geqslant \operatorname{Difficulty}\left(\mathcal{X}_{i}\right)$ and

3. $\forall \mathcal{X}_{i} \in \sigma: \mathcal{P}_{\text {time }} \geqslant \operatorname{Time}\left(\mathcal{X}_{i}\right)$.

Definition 5 (Profile satisfying attack suite). A profile satisfying attack suite $\sigma$ is a satisfying attack suite which satisfies all the constraints of the chosen attacker profile $\mathcal{P}_{f}$.

\subsection{Description of the approach}

The analysis method can be described by the following rules [10]:

1. The attacker constructs the attack tree and evaluates the parameters of each of the elementary attacks following these considerations:

- The attacker has to spend Cost $_{i}$ resources to prepare and launch an attack $\mathcal{X}_{i}$.

- The attack $\mathcal{X}_{i}$ succeeds with probability $p_{i}$ and fails with probability $1-p_{i}$.

- Depending on the detective security measures, the attacker sometimes has to carry additional costs after failing or succeeding with the attack. The sum of preparation and additional costs is denoted as Expenses $i$ parameter. 
- Additionally, there is global parameter Profit for the whole attack scenario, which describes the benefit of the attacker, in case the root node is achieved.

2. The attacker considers all potential attack suites - subsets $\sigma \subseteq \mathcal{X}$, where $\mathcal{X}=\left\{\mathcal{X}_{1}, \ldots, \mathcal{X}_{n}\right\}$ is the set of all elementary attacks considered in the attack scenario. Some of the attack suites satisfy the Boolean function $\mathcal{F}$, some do not. For the satisfying attack suites the attacker computes the outcome value Outcome . $_{\text {. }}$

3. Finally, the attacker chooses the most profitable attack suite and launches the corresponding elementary attacks simultaneously.

The computational method presented in [10] aims at maximizing the expression

$$
\text { Outcome }_{\sigma}=p_{\sigma} \cdot \text { Gains }-\sum_{\mathcal{X}_{i} \in \sigma} \text { Expenses }_{i}
$$

over all the assignments $\sigma \subseteq \mathcal{X}$ that turn the monotone Boolean function $\mathcal{F}$ to true. The success probability of the primary threat $p_{\sigma}$ can be computed in time linear in the size of elementary attacks $n$ :

$$
p_{\sigma}=\sum_{\substack{\mathcal{R} \subseteq \sigma \\ \mathcal{F}(\mathcal{R}:=\text { true })=\text { true }}} \prod_{\mathcal{X}_{i} \in \mathcal{R}} p_{i} \prod_{\mathcal{X}_{j} \in \sigma \backslash \mathcal{R}}\left(1-p_{j}\right) .
$$

In order to tackle the potential exponential amount of computations in (1), a genetic algorithm was proposed and benchmarked by Jürgenson et al. in [1].

\subsection{Approximation}

The ApproxTree+ method uses the genetic algorithm to facilitate the usage of the computational method for large attack trees:

1. Create the first generation of $n$ individuals (profile satisfying attack suites, not all of them are necessarily distinct).

2. All the individuals in the initial population are crossed with everybody else producing $\left(\begin{array}{l}n \\ 2\end{array}\right)$ new individuals.

3. Each individual is mutated with probability $p$.

4. The mutated population is joined with the initial population.

5. Finally, $n$ fittest profile satisfying individuals out of the $\left(\begin{array}{l}n \\ 2\end{array}\right)+n$ individuals are selected and form the next generation.

The reproduction phase terminates when $k$ last generations do not increase outcome. The complexity of the suggested approach was measured to be approximately $\mathcal{O}\left(0.85^{n}\right)$ using exponential regression. 


\section{Performance analysis}

In order to assess the performance of the introduced computational method we have randomly generated a set of attack trees. The attack tree generation procedure was a two-step process. First, the random Boolean function with the predefined number of variables (leaves in the attack tree) was generated. It contained from 2 to 5 operands per operator - the values of operands in each case were chosen randomly. The next step was to provide quantitative annotations on the leaves of the attack tree. These values were chosen randomly from the predefined intervals: the cost parameter was estimated in the interval $[100,1000]$, the success probability parameter was estimated in the interval $(0,1)$. The value for the difficulty parameter was chosen from uniformly distributed values low, medium, high, and very high. The value for the time parameter was chosen from uniformly distributed values seconds, minutes, hours, and days ${ }^{2}$.

One of the questions that needs to be answered is if attacker profiling adds extra computational overhead. It can be seen on the cumulative time distribution diagram (see Fig. 1) that attacker profiling does not add any significant computational overhead (in the case of a single attacker profile being analyzed) compared to the ApproxTree approach (see Fig. 2). In both methods the initial population generation phase is almost immediate, as well as the mutation phase. The main workload is performed by the crossover phase and consumes approximately $85-99 \%$ of the cumulative time distribution among all the phases. The last phase, the best individuals selection phase, does not introduce any significant workload and consumes approximately $1-15 \%$. The crossover phase is the most time consuming as each individual is crossed with every other individual in the population producing $\mathcal{N} \times \mathcal{N}$ cross operations, where $\mathcal{N}$ is the amount of individuals in the initial population. Fig. 3 shows that the execution time of the ApproxTree+ approach is proportional to the ApproxTree approach. The increased execution time arises from the fact that, as a rule, one doesn't assess risks using just a single adversarial profile, as it is reasonable to assess risks using the entire set of possible adversarial profiles so that the results would produce meaningful insight on the risk landscape - thus the overall execution time is proportional to the number of the attacker profiles under consideration.

The analysis of the speed of convergence shows that the convergence speed of ApproxTree does not exceed the convergence speed of ApproxTree+. Additionally, it does not depend on the size of the attack tree -

\footnotetext{
${ }^{2}$ assuming uniform distributtion of the PRNG output
} 


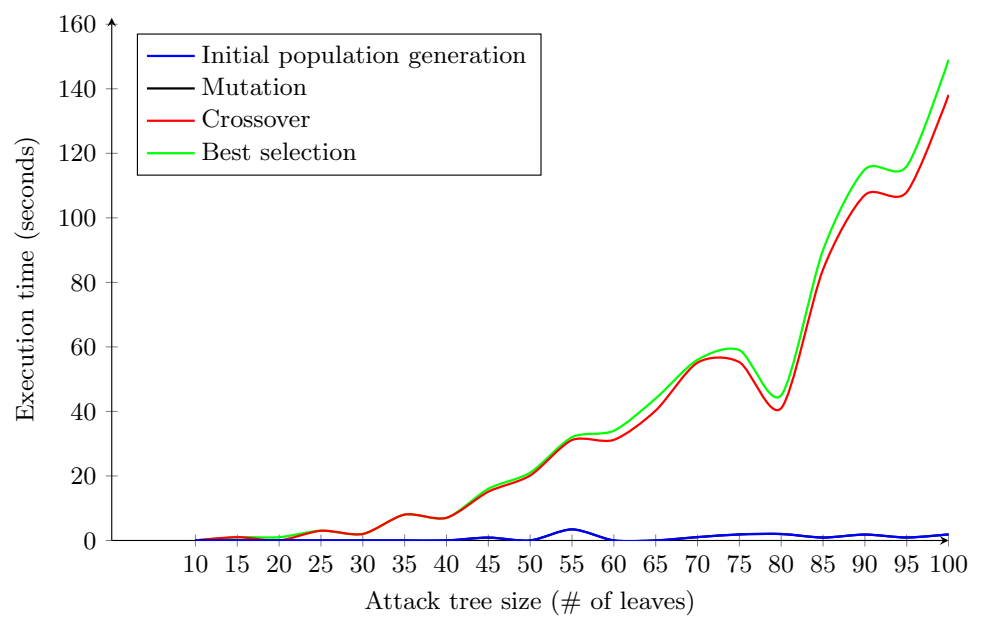

Fig. 1. Cumulative time distribution of ApptoxTree+ phases.

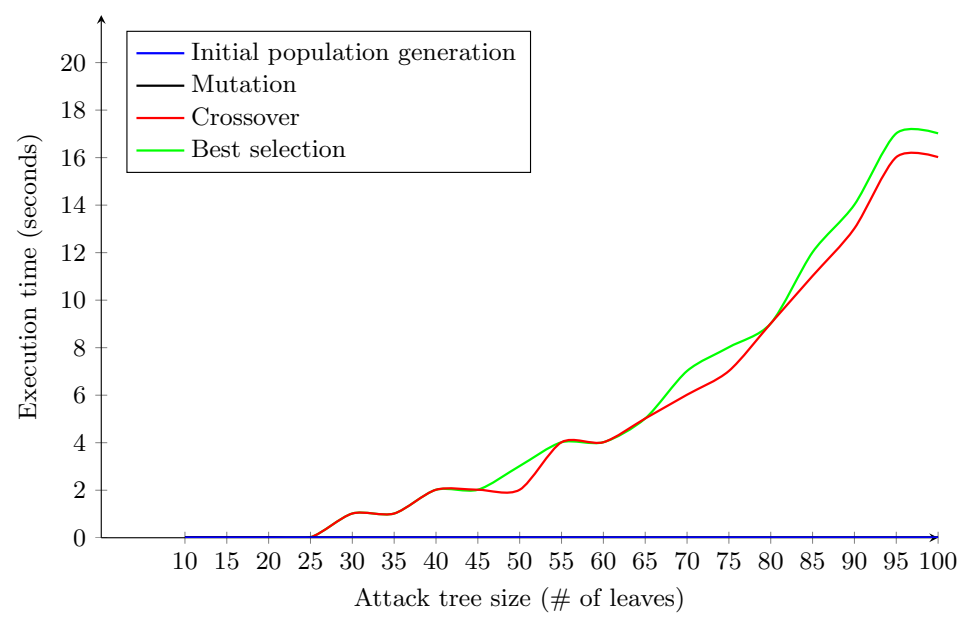

Fig. 2. Cumulative time distribution of ApproxTree phases.

independently of the size of the tree, the convergence speed stays approximately at the same level.

Additionally, we have analyzed the effect of the genetic algorithm parameters such as mutation rate and initial population size on the convergence speed to assess whether the parameters of the genetic algorithm used by ApproxTree [1] are optimal for the ApproxTree+ approach.

The convergence speed decreases with the increase in the percentage of mutations from approximately 2 generations in the case when the mu- 


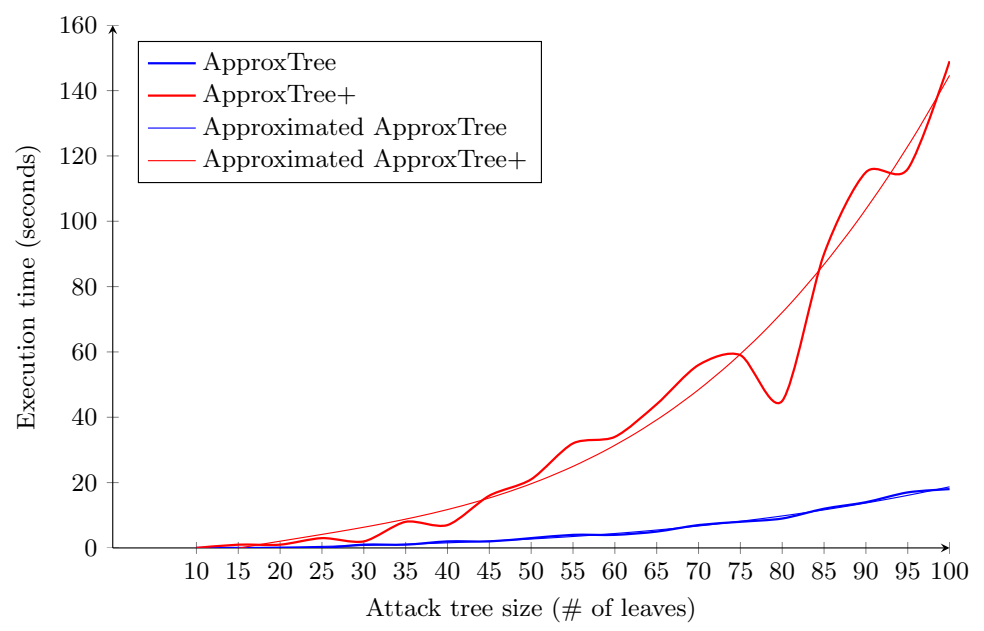

Fig. 3. Execution time.

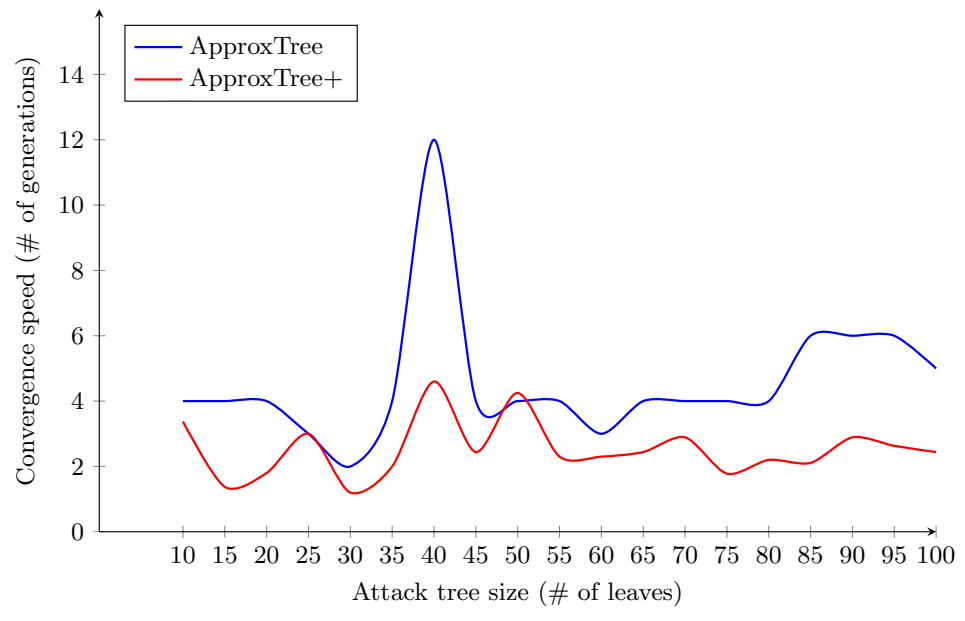

Fig. 4. Convergence speed.

tation rate is $10 \%$ up to 6 generations in the case when mutation rate is 90\% (see Fig. 5). Independently of the mutation rate, the speed of convergence of ApproxTree+ does not exceed the speed of convergence of ApproxTree.

Benchmarking results have shown that the mutation step has no significant effect on the convergence speed at all. We were unable to find any case where the method would get stuck in the local optimum. Even when the mutation step was excluded entirely (as a phase of the genetic 


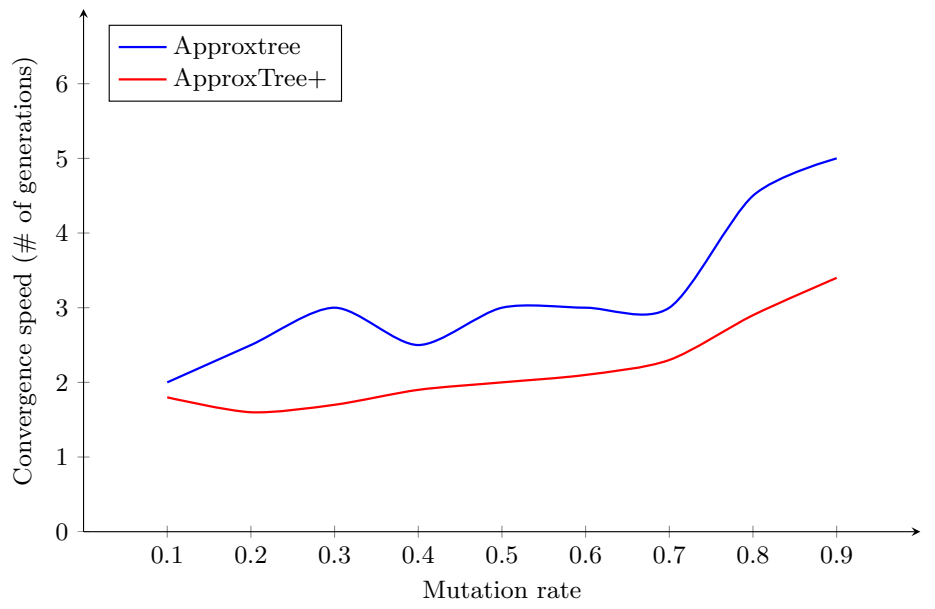

Fig. 5. Convergence speed as a function of the mutation rate.

algorithm) - the global optimum was always reached. This may happen because of "good" initial population generation - if the size of the initial population is rather big compared to the number of satisfying solutions, it is highly likely that the initial population will contain all the solutions (profile satisfying attack suites). In this case the convergence is immediate, which was observed in some cases during benchmarking. If the initial population does not contain all the solutions, still it may be "good enough" so that the crossover step produces the entire domain of solutions.

With the increase in the initial population size (see Fig. 6) the convergence speed increases, stabilising at a value of approximately 1.6 generations for the initial population size greater than $4 n$ ( $n$ being the number of leaves in the attack tree) in the case of the ApproxTree approach. In the case of ApproxTree+ we can see slight, but firm decrease in the convergence speed. In some cases when initial population size was less than $n$ the computational method was unable to reach global optimum, which may happen when rather small initial population limits the amount of possible solutions that may be reached and the mutation rate is small enough and does not improve the situation.

The precision assessment shows that in ApproxTree+, as well as in ApproxTree, either the result converges to the global optimum (most profitable attack suite) or the computational method fails to generate the initial population of individuals. In case of profiling, the attacker profile may contain so strict constraints that not a single profile satisfying attack 


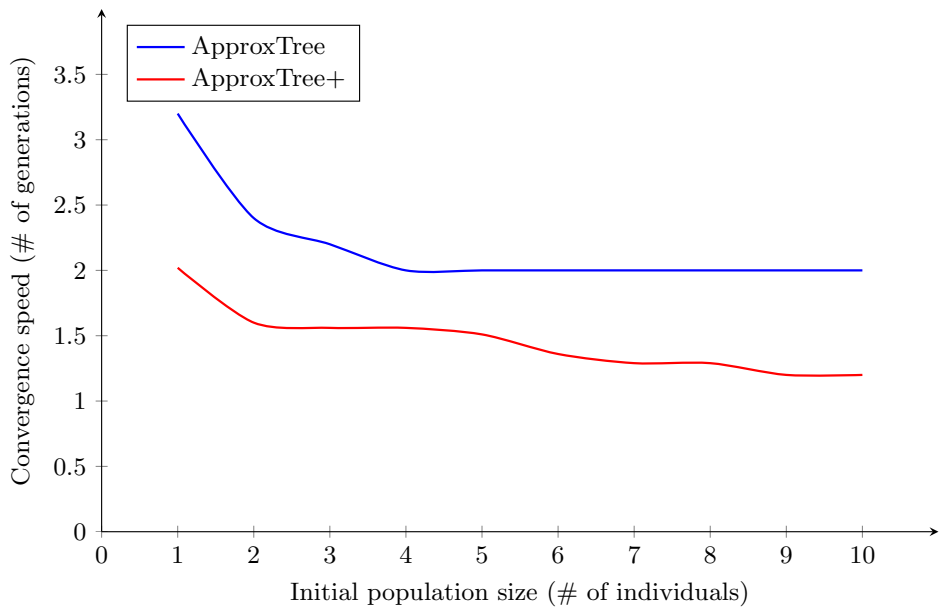

Fig. 6. Convergence speed as a function the size of the initial population.

suite may exist. The more strict constraints are used in the considered attacker profiles the higher is the probability that no profile satisfying assignments will be generated. However we are unable to state that the profile satisfying solutions definitely do not exist in this case, as the state when ApproxTree+ is unable to generate the initial population means 2 possible conditions - either no profile satisfying attack suites exist (and thus the considered attack scenario has no profitable solutions), or such attack suites exist, however the attack suite generation procedure failed to generate profile satisfying solutions due to the stochastic nature of the process.

\section{Conclusions and Future Research}

Attacker profiling is a way to separate infrastructure properties and the properties of the malicious agents who are undertaking strategic decisions in the target infrastructure. This kind of separation allows to estimate and assess these properties independently from one another. This allows to derive meaningful values for the quantitative annotations on the attack steps in complex multi-step attacks from the underlying properties instead of providing joint estimations to these values directly. One can more precisely estimate how would a complex value change in case when some of its underlying components change. In example, how would the likelihood of success in an attack step change if instead of profit-oriented malicious individuals we face organized groups of attackers or a national 
security agency and the target infrastructure was patched meanwhile and the employees have received an awareness training? Thus, attacker profiling enables more detailed assessment of the impact of the fluctuations in threat and vulnerability landscapes on the values of the quantitative annotations on the attack steps.

Additionally, it adds flexibility to the analysis in general, enabling analysis using different combinations of attacker profiles and infrastructure properties, making comprehensive risk assessment possible. It provides broader and more detailed overview of the risk landscape in a timely manner, following constant changes in the risk environment. It allows to make informed decisions in assessing the cost-effectiveness of the defensive measures and enabling the prediction, prioritization and prevention of emerging attacks in nearly semi-automated way.

The separation between the attacker and infrastructure properties was implemented in the attack navigator, one of the innovations of the $\mathrm{TRE}_{\mathrm{S}} \mathrm{PASS}$ project [32]. It consists of a navigator map describing the infrastructure, and a set of attacker profiles, representing malicious agents in the considered environment traversing the map. Various strategic preferences of malicious agents acting in this environment result in different paths that these agents follow to achieve their goals thus allowing to derive attack vectors that the considered types of attackers would prefer. Additionally, the process of generating attack scenarios from socio-technical security models was elaborated, automating the process of assembly of such scenarios. We see attacker profiling as a step forward in tackling challenges of the security metrics.

We introduced the attacker profiling and demonstrated the application of profiling in the framework of attack tree analysis by introducing the new analysis tool named ApproxTree+ and demonstrating that integrating attacker profiling into an existing analysis method does not introduce any significant performance penalty.

The constraint based approach, outlined in the paper, is only one possible interpretation of attacker profiling. Another possibility is to apply Item Response Theory to represent the relation between various underlying components in the threat and vulnerability landscapes. Such a relation may be represented, in example, in the form of a logistic function in its simplest form indicating that the likelihood of success will be assigned value 0.5 when the skill $(\beta)$ and difficulty $(\gamma)$ are equal: $p=\left(e^{\beta-\delta}\right) /\left(1+e^{\beta-\delta}\right)$. In more complex scenarios the function may be extended to take 3 arguments, as the likelihood of success depends on the 
invested time parameter as well, and in this case it will take the form of: $p=f(\beta, \delta, \gamma)$ where $\gamma$ is the time invested into attacking.

We see the way forward in implementing the above mentioned interpretation of profiling, integrating ApproxTree+ in the existing risk assessment frameworks and tools, and validating the approach in real-case risk analysis.

\section{References}

1. Jürgenson, A., Willemson, J.: On fast and approximate attack tree computations. In Kwak, J., Deng, R.H., Won, Y., Wang, G., eds.: ISPEC. Volume 6047 of Lecture Notes in Computer Science., Springer (2010) 56-66

2. Vesely, W.E., Goldberg, F.F., Roberts, N.H., Haasl, D.F.: Fault Tree Handbook. U.S. Nuclear Regulatory Commission, Washington, DC (1981)

3. Schneier, B.: Attack trees. Dr. Dobb's Journal of Software Tools 24(12) (December 1999) 21-22, 24, 26, 28-29

4. Schumacher, M.: Security Engineering with Patterns - Origins, Theoretical Models, and New Applications. Volume 2754 of Lecture Notes in Computer Science. Springer (2003)

5. Miede, A., Nedyalkov, N., Gottron, C., König, A., Repp, N., Steinmetz, R.: A generic metamodel for it security. In: ARES, IEEE Computer Society (2010) 430437

6. Kishor S. Trivedi, Dong Seong Kim, A.R., Medhi, D.: Dependability and security models. In: Proceedings of the 7th IEEE International Workshop on the Design of Reliable Communication Networks (DRCN), Washington, DC (October 2009) $11-20$

7. Schneier, B.: Secrets \& Lies: Digital Security in a Networked World. 1st edn. John Wiley \& Sons, Inc., New York, NY, USA (2000)

8. Kordy, B., Mauw, S., Radomirović, S., Schweitzer, P.: Attack-Defense Trees. Journal of Logic and Computation 24(1) (2014) 55-87

9. Buldas, A., Laud, P., Priisalu, J., Saarepera, M., Willemson, J.: Rational choice of security measures via multi-parameter attack trees. In López, J., ed.: CRITIS. Volume 4347 of Lecture Notes in Computer Science., Springer (2006) 235-248

10. Jürgenson, A., Willemson, J.: Computing exact outcomes of multi-parameter attack trees. In Meersman, R., Tari, Z., eds.: OTM Conferences (2). Volume 5332 of Lecture Notes in Computer Science., Springer (2008) 1036-1051

11. Jürgenson, A., Willemson, J.: Serial model for attack tree computations. In Lee, D., Hong, S., eds.: ICISC. Volume 5984 of Lecture Notes in Computer Science., Springer (2009) 118-128

12. Mauw, S., Oostdijk, M.: Foundations of attack trees. In Won, D., Kim, S., eds.: ICISC. Volume 3935 of Lecture Notes in Computer Science., Springer (2005) 186198

13. Buldas, A., Stepanenko, R.: Upper bounds for adversaries' utility in attack trees. In Grossklags, J., Walrand, J.C., eds.: GameSec. Volume 7638 of Lecture Notes in Computer Science., Springer (2012) 98-117

14. Buldas, A., Lenin, A.: New efficient utility upper bounds for the fully adaptive model of attack trees. In Das, S.K., Nita-Rotaru, C., Kantarcioglu, M., eds.: GameSec. Volume 8252 of Lecture Notes in Computer Science., Springer (2013) $192-205$ 
15. Kordy, B., Pietre-Cambacedes, L., Schweitzer, P.: Dag-based attack and defense modeling: Don't miss the forest for the attack trees. CoRR abs/1303.7397 (2013)

16. Phillips, C., Swiler, L.P.: A graph-based system for network-vulnerability analysis. In: Proceedings of the 1998 Workshop on New Security Paradigms. NSPW '98, New York, NY, USA, ACM (1998) 71-79

17. series, K.Y.E.: Honeynet project. know your enemy the tools and methodologies of the script kiddie. http://project.honeynet.org (jul 2000)

18. series, K.Y.E.: Honeynet project. know your enemy ii: Tracking the blackhat's moves. http://project.honeynet.org (jun 2001)

19. series, K.Y.E.: Honeynet project. know your enemy iii: They gain root. http://project.honeynet.org (mar 2000)

20. Blomquist, A., Arvola, M.: Personas in action: ethnography in an interaction design team. In: Proceedings of the second Nordic conference on Human-computer interaction. NordiCHI '02, New York, NY, USA, ACM (2002) 197-200

21. Castro, J.W., Acuña, S.T., Juzgado, N.J.: Integrating the personas technique into the requirements analysis activity. In Gelbukh, A.F., Adiba, M.E., eds.: ENC, IEEE Computer Society (2008) 104-112

22. Faily, S., Flechais, I.: Barry is not the weakest link: eliciting secure system requirements with personas. In McEwan, T., McKinnon, L., eds.: BCS HCI, ACM (2010) 124-132

23. Faily, S., Flechais, I.: Persona cases: a technique for grounding personas. In Tan, D.S., Amershi, S., Begole, B., Kellogg, W.A., Tungare, M., eds.: CHI, ACM (2011) 2267-2270

24. Quarantelli, E.L.: The structural problem of a sociological speciality : collective behavior's lack of a critical mass. The American sociologist (1974) A version of the article was presented at the annual meeting of the American Sociological Association in New York City, 30.8.1973.

25. Marx, G.T., Wood, J.L.: Strands of theory and research in collective behavior. Annual Review of Sociology 1(1) (1975) 363-428

26. Chau, M., Xu, J.: Mining communities and their relationships in blogs: A study of online hate groups. Int. J. Hum.-Comput. Stud. 65(1) (January 2007) 57-70

27. Chen, P.: Imitation, learning, and communication: Central or polarized patterns in collective actions. In Babloyantz, A., ed.: Self-Organization, Emerging Properties, and Learning. Volume 260 of NATO ASI Series. Springer US (1991) 279-286

28. Dinev, T., Hu, Q.: The centrality of awareness in the formation of user behavioral intention toward protective information technologies. J. AIS 8(7) (2007)

29. Pardue, H., Landry, J., Yasinsac, A.: A risk assessment model for voting systems using threat trees and monte carlo simulation. In: Requirements Engineering for e-Voting Systems (RE-VOTE), 2009 First International Workshop on. (2009) 5560

30. Sallhammar, K., Knapskog, S.J., Helvik, B.E.: Building a stochastic model for security and trust assessment evaluation. http://q2s.ntnu.no/publications/open/2005/Mass_media/2005_sallhammar_BSM.pdf (oct 2005)

31. Tipton, H., Baker, P.: Official (isc)2 guide to the cissp cbk. In: Official (ISC)2 guide to the CISSP CBK. (2010)

32. FP7, E.: The trespass project. http://trespass-project.eu (nov 2010) 